\title{
THE DESIGN-FIX FOR FIXED-VALVE MICROPUMPS
}

\author{
Christopher J. Morris and Fred K. Forster \\ Department of Mechanical Engineering, University of Washington \\ Seattle, WA 98195 USA
}

\section{INTRODUCTION}

The deceptively simple fixed-valve micropump concept has been investigated for over five years for both Teslatype and diffuser valve geometries $[2,4]$. Although some of the first steady-flow experiments indicate the Teslatype valve has better valve characteristics (higher diodicity), a review of the literature shows the operation of this type of pump is highly dependent on many factors in addition to the valve design, a number of which are still not understood well enough to fully exploit potential applications. Because of the significant potential of this design for 1) handling liquids containing large particles, 2) reliability and 3) scalability, it is important to utilize a rational design approach that investigates all significant parameters affecting performance. While modeling has shown that a high-Q resonant behavior is essential for good performance [1], attempts to predict performance before fabrication efforts have not been successful [4]. We present here our latest results in the three critical areas of design: 1) reduced order modeling, 2) mechanical/piezoelectric behavior and 3) valve fluid mechanics. Through careful design it is possible to develop a goodperforming pump even with valves that regurgitate $90 \%$ of each delivered pump stroke.

\section{MODELING AND ANALYSIS}

Reduced Order Modeling: A linear system graph of an entire pump is shown in Fig. 1. It reveals how the electrical input on the left is coupled through the mechanical portion of the system in the middle to the fluid components shown on the right. This representation is utilized for 1) linear analysis of the pump to optimize resonant behavior and 2) nonlinear analysis that includes more complete valve characteristics to predict actual pressure/flow performance. The thirteen parameters shown in Fig. 1 are in turn functions of more parameters related to the geometry and material properties of both the structure and fluid. Yet with the use of finite element structural analysis and fluid dynamic calculations, the only input parameters to the model are those fundamental parameters.

Mechanical/Piezoelectric Behavior: We have recently solved the general problem of optimization of the circular bimorph actuator including a bonding layer [3] and have applied the same technique to the case of a square bimorph which we now use for our micropumps, as shown in Fig. 2. This approach utilized the high-level scripting language of ANSYS to perform optimization.



Figure 1. Reduced-order system model for a fixed valve micropump, with the following symbol definitions: $f_{e}$, displacement-per-volt $V ; k$, spring constant; $m$, mass; $\gamma A_{c}$, membrane shape factor times area; $I_{c}$, chamber inductance; $C_{c}$, chamber capacitance; $I_{v i}$ and $I_{v o}$, inlet/outlet valve inductance; $R_{v i}$ and $R_{v o}$ inlet/outlet valve resistance; $C_{i}$ and $C_{o}$, inlet/outlet capacitors.

The analysis provides accurate values for displacementper-volt, swept volume and membrane spring constant needed in the reduced order model (see Fig. 3).

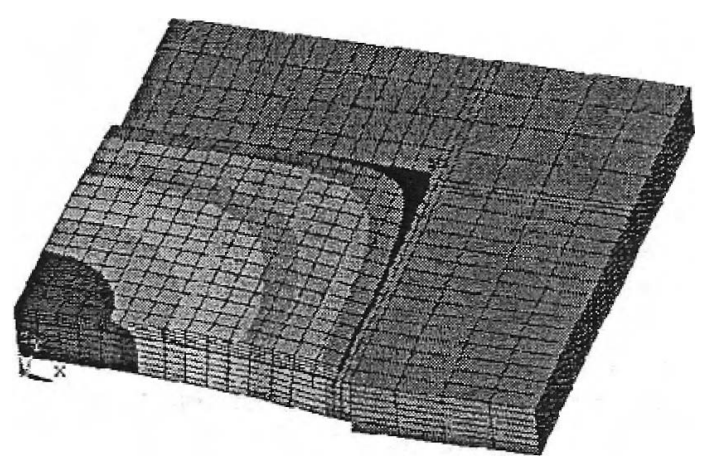

Figure 2. A three-dimensional finite element model of one-quarter of a square micropump membrane, showing the solution for transverse displacement.

Valve Fluid Mechanics: Fluid parameters of the valves including resistance $R$, inductance $I$, and diodicity are as important as any of the structural parameters. Here we present one important aspect of $R$ and $I$ which has been overlooked in numerous investigations, and can be a major source of error in predicting pump resonant behavior. We have determined that the full transient solution of oscillating viscous flow in the valves is required to properly model valve behavior in terms of hydraulic 


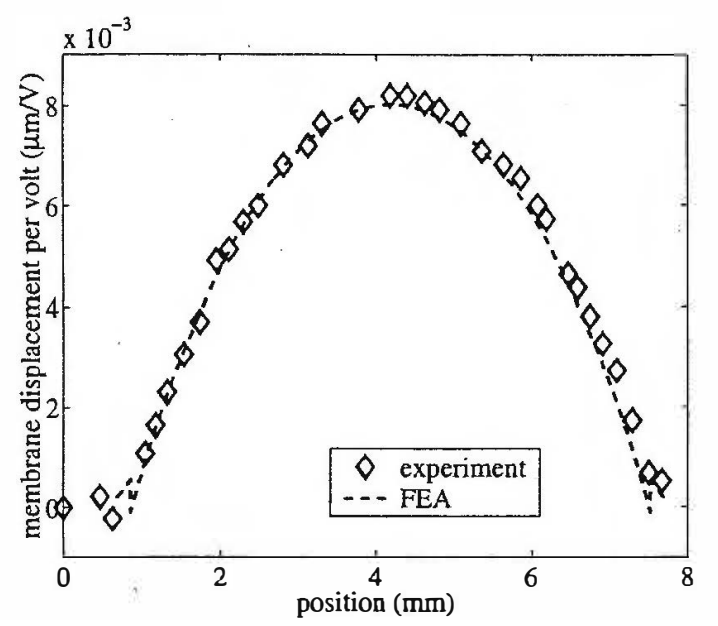

Figure 3. Static displacement-per-volt of a $7 \mathrm{~mm}$ square pump membrane compared to the FEA model prediction.

impedance $Z=R+i \omega I$. We emphasize this point in this study by presenting results for a simple rectangular duct. The commonly-used expression for $R$ is based on steady viscous flow, and the commonly-used expression for $I$ is based on unsteady inviscid flow. These expressions lead to a simplified, ad hoc hydraulic impedance $Z_{s}$ that differs by as much as $30 \%$ near the cut-off frequency when compared to the exact impedance $Z_{\boldsymbol{e}}$, derived from the Navier-Stokes equations. When applied to a resonant micropump, the ad hoc $Z_{s}$ led to a prediction that was in error by as much as $300 \%$. This result is shown in Fig. 4 using a chamber/channel system that was similar to a fixed-valve micropump, except that the non-linear valve elements were replaced by straight channels, for which $Z_{\boldsymbol{e}}$ could be calculated directly. The striking agreement with experiment with simple channels clearly indicates the modeling improvement based on the exact solution.

By applying the exact impedance of a rectangular channel to a channel-segment, series-parallel model of the Tesla-type valve, the resonant behavior of an actual micropump was predicted. This is shown in Fig. 5, along with what the prediction would be without the full viscous solution. Reasonable agreement was obtained, which allows the use of the nonlinear model to optimize net pump output pressure and flow. More recent results indicate that differences seen between experiment and model results in Figs. 5 and 4 can be addressed through computational modeling of the transient flow in the valves.

\section{REFERENCES}

[1] R L Bardell et al. Designing high-performance micropumps based on no-moving-parts valves. In $M E M S$, volume DSC-Vol.62/HTD-Vol.354. ASME IMECE, 1997.

[2] F K Forster et al. Design, fabrication and testing of fixed-valve micro-pumps. In ASME Fluids Div., volume 234. ASME IMECE, 1995.

[3] C. J. Morris and F. K. Forster. Optimization of a circular piezoelectric bimorph for a micropump driver. Accepted for publication in J. Micromech. Microeng., 2000.

[4] A. Olsson et al. A numerical design study of the valveless diffuser pump using a lumped-mass model. J. Micromech. Mircoeng., 9, 1999.

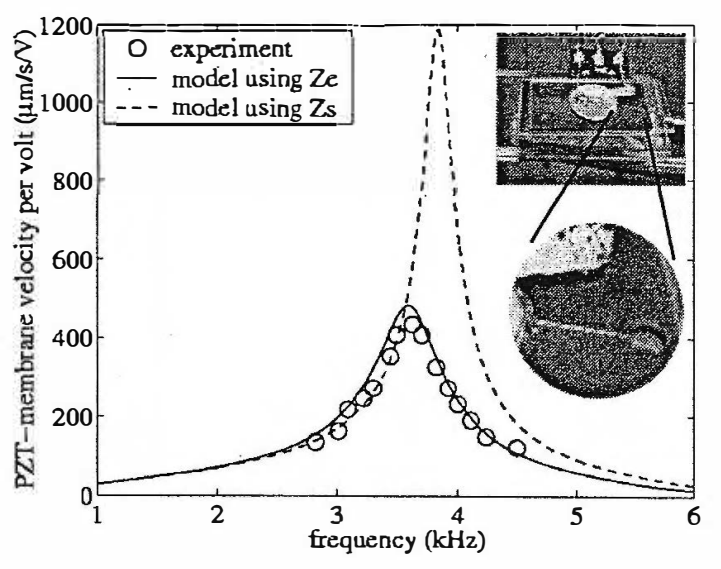

Figure 4. Membrane velocity frequency response of the chamber/channel system shown in the upper right corner, and comparison with model predictions based on simplified and exact impedance, $Z_{s}$ and $Z_{e}$. Shown is good agreement with experiment when the exact form of impedance $Z_{e}$ is utilized, and over-prediction by $300 \%$ for the commonly-used method based on $Z_{s}$.

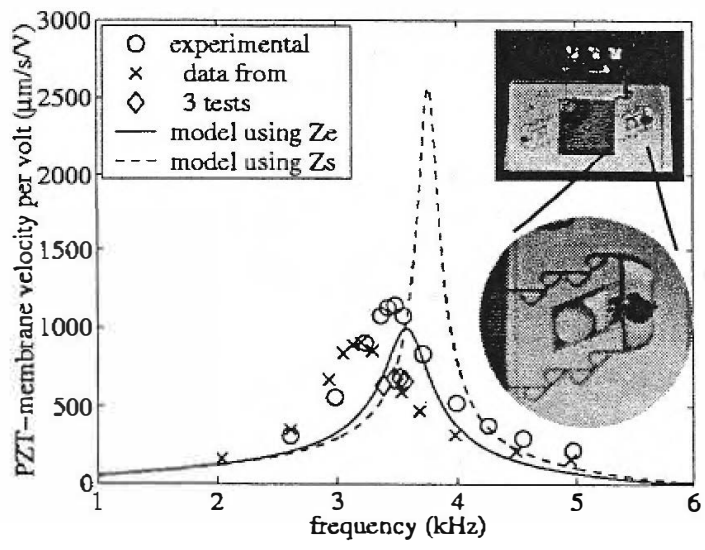

Figure 5. Membrane velocity frequency response of the square-chamber micropump system shown in the upper right corner, and comparison with model predictions based on simplified and exact impedance, $Z_{s}$ and $Z_{e}$. Shown is good agreement with experiment when the exact form of impedance $Z_{\boldsymbol{e}}$ is utilized, and over-prediction by $300 \%$ for the commonly-used method based on $Z_{s}$. 